\title{
Voxel-based diagnosis of Alzheimer's disease using classifier ensembles
}

\author{
Rubén Armañanzas, Martina Iglesias, Dinora A. Morales, and
}

\begin{abstract}
Functional magnetic resonance imaging (fMRI) is one of the most promising non-invasive techniques for early Alzheimer's disease (AD) diagnosis. In this paper, we explore the application of different machine learning techniques to the classification of fMRI data for this purpose. The functional images were firstly preprocessed using the statistical parametric mapping toolbox to output individual maps of statistically activated voxels. A fast filter was applied afterwards to select voxels commonly activated across demented and non-demented groups. Four feature ranking selection techniques were embedded into a wrapper scheme using an inner-outer loop for the selection of relevant voxels. The wrapper approach was guided by the performance of six pattern recognition models, three of which were ensemble classifiers based on stochastic searches. Final classification performance was assessed from the nested internal and external cross-validation loops taking several voxel sets ordered by importance. Numerical performance was evaluated using statistical tests, and the best combination of voxel selection and classification reached a $\mathbf{9 7 . 1 4 \%}$ average accuracy. Results repeatedly pointed out Brodmann regions with distinct activation patterns between demented and non demented profiles, indicating that the machine learning analysis described is a powerful method to detect differences in several brain regions between both groups.
\end{abstract}

Index Terms-Machine learning, Supervised classification, Feature selection, Alzheimer's disease, fMRI.

\section{INTRODUCTION}

A LZHEIMER'S disease (AD) is a neurodegenerative disorder that affects brain regions related to cognitive functions such as memory, attention, language and problem solving. Being the most common form of dementia, AD is suffered by $10 \%$ of people aged over 65 years and nearly $50 \%$ of the population aged over 85 . Worldwide, nearly 44 million people have Alzheimer's or a related dementia (as estimated by Alzheimer's Disease International [1]).

In this paper, we explore the application of different supervised classification paradigms to the study of functional magnetic resonance imaging (fMRI) data produced by a clinical study of AD patients and healthy controls [2]. This data has the potential to construct prediction systems for decoding intentions or perceptual states, as well as aiding tools to discriminate healthy from potentially affected subjects. The

R. Armañanzas is with the Krasnow Institute for Advanced Study, George Mason University, Fairfax, VA 22030, USA e-mail: (see http://mason.gmu. edu/ rarmanan).

M. Iglesias and D.A. Morales were with Centro de Supercomputación de Madrid, Universidad Politécnica de Madrid, 28660 Boadilla del Monte, Madrid, Spain.

L. Alonso-Nanclares is with the Instituto Cajal, CSIC, Avenida Doctor Arce 37, 28002 Madrid, Spain; and with the Laboratorio Cajal de Circuitos Corticales, Centro de Tecnología Biomédica, 28223 Pozuelo de Alarcón, Spain. literature on automatic classification systems based on fMRI is depth and breadth. From deception detection using fMRI feature selection [3] to emotional analysis of synesthetes [4], the apparently endless list of neurological applications of this technology is growing all the time [5]. Regarding AD in particular, MRI-based automatic classification of scans in $\mathrm{AD}[6]$ or advances in the diagnosis of $\mathrm{AD}$ patients using fMRI-derived features [7] have been proposed.

We here propose the direct use of fMRI voxel profiles of elderly brains to tackle the automatic pattern analysis of $\mathrm{AD}$ and healthy individuals. Hence, this research aims at two key objectives. First, a spatial localization aims to address brain regions of interest when comparing $\mathrm{AD}$ and control activation profiles. Secondly, a pattern discrimination utility, i.e., use of the differentially activated voxels from the detected regions of interest to induce a support-decision system able to discriminate $\mathrm{AD}$ from control subjects. In terms of data analysis, the challenge focuses on the dimensionality problem and how feature subset selection is helpful for dealing with the well-known problem of the curse of dimensionality [8]. To overcome it, we propose the joint combination of four different voxel selection techniques with six pattern recognition algorithms, giving prominence to ensemble approaches. These ensemble methods are based on random searches, which help combat fMRI data instability and the overfitting of classical models when redundant or noisy features are present. As we consider classifier prediction performance as an indicator of informative voxel subsets, it is advisable to use different selection methods. Results from the pattern discrimination stage include lists of relevant voxels and their performance assessment estimated by means of fair techniques, such as inner-outer loop error estimation. The spatial localization of the most relevant subsets is discussed in terms of the degree of affection of the brain areas to which those voxels belong.

The paper is organized as follows. Section III presents the data, voxel selectors and classification models. Both quantitative and qualitative results are reported throughout Section III with spatial plots of the most relevant selected voxels. Section IV discusses the estimated classification performances, as well as the neurological significance of the identified voxels. Finally, Section $\mathrm{V}$ summarizes conclusions and future work strands.

\section{MATERIALS AND METHODS}

\section{A. Data preprocessing}

The chosen dataset is sourced from the fMRI Data Center maintained at Dartmouth College (Hanover, $\mathrm{NH}$ ) and it is 
composed of both structural and functional scans from 41 right-handed English-speaking individuals. Although the cohort includes three different experimental groups, only elderly groups were included to avoid bias in the results due to age differences. The first group, labeled as Group ND, consisted of fourteen non-demented elderly subjects (five males) recruited from the registry of the Washington University Alzheimer's Disease Research Center (ADRC). The ages ranged from 66 to 89 years old (mean age 74.9). The second group, labeled as Group D, consisted of thirteen subjects (six males) between 68 and 83 years old (mean age 77.2) with very mild (8 subjects) to mild dementia (5 subjects) of the Alzheimer's type (DAT). Participants of Group D were also recruited from the Washington University ADRC. Aged subjects were clinically assessed using the Clinical Dementia Rating (CDR) index, where a CDR value of 0 is equivalent to no dementia, whereas CDR values of 0.5 and 1 prescribe very mild and mild DAT, respectively. For further details on the inclusion criteria see [2]. Raw data was deposited in the National fMRI Data Center (http://www.fmridc.org) under accession number 2-2000-1118w. Alternately, data is available upon request (14 GB zipped file).

Functional images were acquired on a Siemens 1.5-Tesla Vision System with an asymmetric spin-echo sequence sensitive to BOLD-contrast using the following parameters: volume $\mathrm{TR}=2.68 \mathrm{sec} ; 3.75 \times 3.75 \mathrm{~mm}$ in-plane resolution; $\mathrm{T} 2 *$ evolution time $=50 \mathrm{msec}$; alpha $=90^{\circ}$. White brain imaging was performed using 16 contiguous 8-mm thick axial oblique slices (acquired parallel to the plane of anterior-posterior commisures).

Scans were produced following a classical visual-motor paradigm test. The basic task paradigm consisted of the presentation of a $1.5-\mathrm{sec}$ duration visual stimulus. Scanning sessions, hereafter referred to as runs, were structured such that one of two kinds of trial condition was presented for each eight-image acquisition $(21.44 \mathrm{sec})$. Participants pressed a key with their right index fingers upon stimulus onset. Functional scans were organized in four runs. Each run included 128 images and lasted approximately 5.5 minutes. A two-minute delay was introduced between runs, during which subjects were permitted to rest. Six images were discarded due to image instability. fMRI data was first preprocessed to remove several sources of noise and artifacts using known procedures from [9]:

1) All functional image runs were normalized to a wholebrain global signal intensity of 1,000 using a single scaling factor per run and slice timing correction was applied to correct the differences in time acquisition between slices.

2) Motion correction using a rigid-body rotation and translation correction were then applied within each functional run and between functional runs within each subject, respectively.

3) All image volumes were spatially normalized to conform to the Montreal Neurological Institute (MNI) atlas [10] and smoothed using a Gaussian kernel of $6 \mathrm{~mm}^{3}$.

For each of the runs, the statistical map of voxel acti- vations was computed using the SPM software [9]. These maps assume that the voxels are distributed according to a known probability Student's $\mathrm{t}$ distribution, and they are commonly referred to as $t$-maps [11]. To create the supervised classification data matrix, each of the four $t$-maps per subject were vectorized in a single dimension array. To avoid false positive activations, only voxels whose activation levels were detected in at least $20 \%$ of the images simultaneously for each phenotype were retained. The final classification matrix included 107 data instances with 4,132 voxels as features. For full detail see Section $\Pi$ III.

\section{B. Machine learning methods}

1) Pattern recognition models: The automatic label assignment of a new sample based on the knowledge provided by a cohort of similar and dissimilar samples is broadly known as pattern recognition. The variable which maps the separation between already known samples is called the supervised variable or class variable. Analyses entailing phenotypic distinction between control and disease samples are broadly known as supervised classification problems.

Formally, a classification dataset $D$ is comprised of a set of $N$ observations (samples or instances), each of which is described by $n+1$ random variables (features). The first $n$ variables, $X_{1}, X_{2}, \ldots, X_{n}$, are known as predictive variables, and the variable in the $n+1$ position is the class variable $C$. Therefore, a classifier can be seen as a function that assigns labels to observations,

$$
\gamma:\left(x_{1}, \ldots, x_{n}\right) \rightarrow\{1,2, \ldots, m\},
$$

where $\boldsymbol{x}=\left(x_{1}, \ldots, x_{n}\right) \in \mathcal{R}^{n}$ is the observation and $\{1,2, \ldots, m\}$ is the range of possible values for the class variable $C$.

In this research, six different pattern recognition models were chosen to tackle the classification task. The first three are regular classification inducers known to work well within fMRI analyses [12]. The other three are metaclassifier schemes -also known as ensembles- based on stochastic searches specially fitted to the sparse nature of fMRI data. There follows a brief summary of each paradigm including details regarding their mathematical groundwork.

a) Support vector machines: (SVMs) have a long history as the most popular classifier for both classification and feature selection in fMRI data analysis. SVMs with linear kernels use a hyperplane as the classification boundary between two classes. This is constructed in such a way that the hyperplane is furthest away from the nearest points of opposite classes. This maximizes the margin of the classifier, which translates into a better generalization performance. The ability of SVM to project data to higher dimensions in the search of linear separability makes them especially suitable for large sized feature spaces. Specifically, sequential minimal optimization (SMO) [13] and LibLinear (large-scale linear support vector regression [14]) algorithms were compared.

b) Naïve Bayes: (NB) is based on and optimal for the assumption of conditional independence between the predictor variables given the class [15]. Although this assumption does 
not hold in this context, we included this model because it has yielded acceptable results in other fMRI studies [12]. The model parameters are estimated with a factorization based on the normal distribution assumption for each predictive variable.

c) Random forests: (RF) is the first of the three ensembles that we have used. Random forests [16] is a version of bagging where the baseline classifier is a modified decision tree, termed random tree. The difference is in the classifier training: a decision tree is a deterministic classifier, whereas a random tree is not. Hence substantially different trees can be constructed from identical training data. We considered forests of 10 random trees of unlimited depth.

d) Random subspace: (RS) is another ensemble method that is composed of several individual classification models [17]. This paradigm works with any model type, and we chose to use SMO because it yielded competitive performance accuracies in preliminary tests. In addition, each classifier in the ensemble is trained on a random subset of features. The subsets can be intersecting or disjoint, and the outputs are aggregated by majority vote. The feature space was divided into 10 subspaces due to data limitations.

e) Random linear oracle: (RLO) also makes use of a SMO as the baseline model in the ensemble [18]. The ensemble part of the oracle is that the training data is randomly divided into two parts by a hyperplane, and separate SMO classifiers are induced for each part. Each ensemble member is itself a mini-ensemble with two classifiers. A new instance for classification is labeled taking into account the output of the classifier assigned to the space region to which the new instance belongs. Outputs are again aggregated by majority vote decision.

2) Voxel subset selection: The law of parsimony states that mathematical models with the smallest number of parameters are preferable, as each parameter introduced into the model adds some uncertainty. In this respect, it has already been proven that the classification accuracy of supervised classification algorithms is not monotonic with respect to the addition of features [19]. The predictive accuracy of such models could be lessened by irrelevant or redundant features.

Therefore, one of the main goals of our analysis was to find subsets of relevant voxels for the induction of classifiers able to help distinguish $\mathrm{AD}$ from control cases. This problem is usually known as feature subset selection and here we will refer to it as voxel subset selection (VSS). VSS is basically a search problem: each state in the search space corresponds to a different configuration for the subset $\boldsymbol{X}^{\prime} \subset \boldsymbol{X}$, and each configuration has an associated objective function value. This objective function measures the goodness of the configuration, e.g. classification performance. Exhaustive evaluation of all possible subsets is unfeasible when there are more than a few dozen voxels [20]. Instead, we opted to measure the goodness or relevance of each individual voxel based on a univariate relevance metric. These univariate metrics output a coefficient that quantifies how relevant a voxel is to the problem.

Each voxel coefficient can be seen as a mark or merit, and they can then all be ordered in a relevance ranking. In this way, a ranking of voxels is computed according to a particular relevance metric. Four different metrics/algorithms were used to compute each relevant ranking. We chose six cut-offs, namely 10, 25, 50, 100, 500 and 1,000 voxels, respectively, in order to limit the number of voxels per ranking, while exploring different cardinalities. There follows a brief description of each method.

a) Random feature elimination: (RFE) is a wrapper feature selection method based on the weights assigned to each feature during SVM induction [21]. It recursively trains SVMs removing the worst ranked features in each step, finally obtaining a feature ranking.

b) ReliefF: ReliefF is a filter feature selection method which uses distance heuristics to search for the best subset of features [22]. A key idea of the ReliefF algorithm is to estimate the goodness of a feature according to how well its values distinguish between similar cases that belong to different classes.

c) Activation sum: It ranks the voxels according to their activation level across all dataset samples. It can be classified as a univariate filter approach [23].

d) Activation per class: Similar to the previous method, it ranks voxels individually for each class also using their associated level of activation [12]. In this case, the output voxel ranking is formed by merging all the intermediate class rankings.

3) Performance assessment: Classical validation schemes such as single train-and-test, cross-validation or 0.632 bootstrap are known to have adverse effects on the final estimators when dealing with feature (voxel) selection procedures [24]. To avoid unwanted effects, the performance assessment reported in this paper followed a nested inner-outer crossvalidation scheme [25]. The outer iteration splits $k-1$ folds as the training set and keeps the remaining fold as the outer test set. After this division, the voxel selection procedures are applied separately to the outer training set only. The voxels with the highest estimated accuracy are then selected by an inner $k$-fold cross-validation procedure and used to assess the outer accuracy estimator. The external loop is repeated $k$ times, and each classification model is then fairly evaluated by its external accuracy, i.e., the average estimator from all outer folds.

The performance of every combination of voxel selection and classifier was assessed according to this scheme and using a value of five folds for both the inner and outer crossvalidations $[25]$.

\section{RESULTS}

The diagnosis of $\mathrm{AD}$ among elderly people (D vs. ND) using fMRI profiles could be a great help for the clinical assessment of potentially demented subjects. In this regard, the confounding effect of the age differences is also removed from the data analysis. This problem represents a realistic application of supervised classification to AD diagnosis in daily clinical practice. The data matrix contained 51 instances of AD subjects (D), four runs per subject except for subject 36 for which there were only three runs; and 56 instances of subjects belonging to the non-demented over-65 group (ND), 
TABLE I

NUMERICAL PERFORMANCE (AVERAGE ACCURACY, STANDARD DEVIATIONS) FOR ALL COMBINATIONS OF CLASSIFIER AND VOXEL SUBSET SELECTORS IN THE DEMENTED $v s$. NON-DEMENTED CLASSIFICATION. COLUMNS AND VALUES HIGHLIGHTED WITH A $\diamond$ SYMBOL ARE THE HIGHEST VALUES ON AVERAGE. THESE HIGHEST VALUES ARE TAKEN AS A BASELINE FOR ASSESSING STATISTICAL DIFFERENCES BETWEEN THEM AND THE VALUES IN THE RESPECTIVE ROWS. THE $\dagger$ SYMBOL MAPS VALUES THAT ARE SIGNIFICANTLY WORSE THAN THE BASELINE COLUMN FOR $\alpha=0.05$, WHEREAS THE $\ddagger$ SYMBOL MAPS SIGNIFICANCE AT A LEVEL OF $\alpha=0.01$. BOLD-FACED VALUES HIGHLIGHT THE BEST INDIVIDUAL COMBINATION OF NUMBER OF VOXELS, CLASSIFIERS AND VOXEL SELECTORS.

\begin{tabular}{|c|c|c|c|c|c|c|}
\hline \multicolumn{7}{|c|}{ Random feature elimination ranking } \\
\hline$\overline{\mathrm{n}}$ & SMO & LibLinear & Naïve Bayes & Random F. & Random S. & Linear O. $^{\diamond}$ \\
\hline 10 & $74.94 \pm 10.45$ & $76.62 \pm 3.06$ & $51.47 \pm 5.71^{\ddagger}$ & $78.44 \pm 6.56$ & $65.32 \pm 8.51^{\dagger}$ & $78.48 \pm 2.42$ \\
\hline 25 & $97.14 \pm 2.33$ & $94.37 \pm 1.95$ & $70.89 \pm 7.97^{\dagger}$ & $95.32 \pm 3.02$ & $70.97 \pm 8.31^{\dagger}$ & $92.38 \pm 8.83$ \\
\hline 50 & $80.43 \pm 7.42$ & $81.26 \pm 8.05$ & $74.68 \pm 7.85$ & $85.93 \pm 6.68$ & $82.29 \pm 8.82$ & $87.75 \pm 7.81$ \\
\hline 100 & $94.33 \pm 4.68$ & $93.42 \pm 4.89$ & $76.49 \pm 8.13^{\dagger}$ & $94.29 \pm 7.00$ & $83.07 \pm 12.29^{\dagger}$ & $97.14 \pm 5.71$ \\
\hline 500 & $95.37 \pm 2.88$ & $94.42 \pm 1.76$ & $75.67 \pm 3.61^{\ddagger}$ & $92.51 \pm 2.32$ & $90.61 \pm 3.08$ & $95.32 \pm 3.01$ \\
\hline 1,000 & $94.46 \pm 3.38$ & $93.55 \pm 4.60$ & $64.50 \pm 7.40^{\ddagger}$ & $93.51 \pm 4.67$ & $95.32 \pm 5.96$ & $94.42 \pm 3.49$ \\
\hline ean & $89.44 \pm 8.52$ & $88.94 \pm 7.21$ & $68.95 \pm 8.79$ & $90.00 \pm 5.99$ & $81.27 \pm 10.40$ & $90.92 \pm 6.29^{\diamond}$ \\
\hline \multicolumn{7}{|c|}{ ReliefF ranking } \\
\hline $\mathrm{n}$ & SMO & LibLinear & Naïve Bayes & Random F. & Random S. & Linear $\mathrm{O} . \diamond$ \\
\hline 10 & $67.49 \pm 9.88$ & $68.31 \pm 7.69$ & $45.15 \pm 16.30^{\dagger}$ & $71.00 \pm 5.61$ & $54.20 \pm 8.11^{\dagger}$ & $71.08 \pm 8.44$ \\
\hline 25 & $74.89 \pm 10.28$ & $74.68 \pm 9.02$ & $54.24 \pm 8.68^{\dagger}$ & $77.62 \pm 6.66$ & $62.68 \pm 13.96$ & $77.75 \pm 9.51$ \\
\hline 50 & $83.19 \pm 3.70$ & $79.38 \pm 4.03$ & $63.60 \pm 15.27$ & $86.92 \pm 4.64$ & $73.83 \pm 12.23$ & $83.11 \pm 6.59$ \\
\hline 100 & $85.89 \pm 5.38$ & $91.60 \pm 1.83$ & $64.33 \pm 10.22^{\ddagger}$ & \pm 5.38 & $79.22 \pm 10.88$ & $91.65 \pm 5.41$ \\
\hline 500 & $93.42 \pm 6.49$ & $92.51 \pm 2.32$ & $60.04 \pm 13.31^{\dagger}$ & $90.61 \pm 5.26$ & $86.02 \pm 6.50$ & $88.79 \pm 4.82$ \\
\hline 1,000 & $88.74 \pm$ & 92.5 & $59.83 \pm 1.84^{\ddagger}$ & 5.41 & $8 \pm 7.14$ & $94.46 \pm 4.53$ \\
\hline an & $82.27 \pm 8.69$ & $83.17 \pm 9.61$ & $57.87 \pm 6.56$ & $84.11 \pm 7.53$ & $74.89 \pm 13.31$ & $84.47 \pm 8.13^{\diamond}$ \\
\hline \multicolumn{7}{|c|}{ Activation sum ranking } \\
\hline$\overline{\mathrm{n}}$ & $\overline{\mathrm{SMO}}$ & LibLinear & Naïve Bayes & Random F. & Random S. & 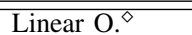 \\
\hline 10 & $72.90 \pm 9.30$ & $72.81 \pm 7.64$ & $64.59 \pm 8.62$ & $73.72 \pm 9.77$ & $75.63 \pm 7.07$ & $74.68 \pm 6.59$ \\
\hline 25 & $83.07 \pm 5.07$ & $82.12 \pm 7.18$ & $46.71 \pm 3.85^{\ddagger}$ & $72.77 \pm 11.24$ & $81.21 \pm 8.67$ & $87.75 \pm 5.82$ \\
\hline 50 & $88.74 \pm 5.71$ & $84.16 \pm 8.68$ & $49.52 \pm 12.18^{\ddagger}$ & $85.06 \pm 6.81$ & $80.35 \pm 6.38$ & $85.97 \pm 5.23$ \\
\hline 100 & $83.20 \pm 13.51$ & $83.16 \pm 11.52$ & $57.97 \pm 11.32^{\ddagger}$ & $85.06 \pm 8.14$ & $76.75 \pm 11.72$ & $84.98 \pm 8.27$ \\
\hline 500 & $88.70 \pm 8.88$ & $90.61 \pm 7.41$ & $53.29 \pm 4.89^{\ddagger}$ & $88.70 \pm 6.41$ & $86.88 \pm 8.22$ & $89.65 \pm 7.05$ \\
\hline 1,000 & $95.28 \pm 5.22$ & $90.65 \pm 7.97$ & $54.11 \pm 15.59^{\ddagger}$ & $=8.23$ & $6 \pm 8.52$ & $91.52 \pm 6.31$ \\
\hline mean & $85.32 \pm$ & $83.92 \pm 6.02$ & $54.37 \pm 5.78$ & $82.50 \pm 6.76$ & $81.90 \pm 5.30$ & $85.76 \pm 5.41^{\diamond}$ \\
\hline \multicolumn{7}{|c|}{ Activation per class ranking } \\
\hline $\bar{n}$ & SMO & LibLinear & Naïve Bayes & Random F. $^{\diamond}$ & Random S. & Linear O. \\
\hline 10 & $77.58 \pm 6.90$ & $77.62 \pm 7.90$ & $53.33 \pm 5.35^{\dagger}$ & $77.58 \pm 10.43$ & $66.36 \pm 11.55$ & $72.03 \pm 9.07$ \\
\hline 25 & $79.30 \pm 7.96$ & $72.71 \pm 7.42$ & $56.69 \pm 15.11$ & $82.07 \pm 10.74$ & $72.71 \pm 8.01$ & $76.60 \pm 6.79$ \\
\hline 50 & $80.35 \pm 6.13$ & $78.57 \pm 6.15$ & $62.77 \pm 8.14^{\dagger}$ & $81.30 \pm 8.63$ & $79.52 \pm 8.80$ & $80.35 \pm 9.38$ \\
\hline 100 & $81.30 \pm 4.19$ & $83.16 \pm 5.59$ & $60.87 \pm 7.49^{\ddagger}$ & $85.15 \pm 6.52$ & $72.99 \pm 7.58$ & $85.15 \pm 5.10$ \\
\hline 500 & $87.25 \pm 10.86$ & $87.16 \pm 8.95$ & $56.48 \pm 21.71^{\dagger}$ & $90.56 \pm 4.35$ & $84.14 \pm 7.64$ & $89.86 \pm 7.27$ \\
\hline 1,000 & $94.45 \pm 3.40$ & $90.64 \pm 3.03$ & $59.63 \pm 12.68^{\dagger}$ & $84.22 \pm 8.51$ & $91.68 \pm 5.31$ & $92.46 \pm 3.87$ \\
\hline mean & $83.37 \pm 5.79$ & $81.64 \pm 6.04$ & $58.29 \pm 3.13$ & $83.48 \pm 3.98^{\diamond}$ & $77.90 \pm 8.32$ & $82.74 \pm 7.18$ \\
\hline
\end{tabular}

four runs $\times 14$ subjects. Each instance contained a total of 4,132 voxels.

We tested four different voxel subset selection methods in combination with six classification paradigms. For each combination, several nested CV experiments were carried out on different voxel subsets as described in Section II-B Quantitative results for all the combinations are shown in Table [ The $\diamond$ symbol in Table I indicates the best classification paradigm on average for each of the voxel selectors. In order to test statistically significant differences, we use the signedrank Wilcoxon test to compare the cross-validation results of every classifier against the baseline. The $\dagger$ symbol in Table I indicates cases where the differences between the value and the baseline are significant for $\alpha=0.05$. Values marked with a $\ddagger$ symbol are significant at a level of $\alpha=0.01$. Since the baseline is selected on the basis of the best average values, significance differences always indicate worse results than the baseline.

Table II summarizes the best classification results. The plots at the top show that the RLO algorithm achieved the best average performance when using RFE, ReliefF and activation sum as selection methods. As for the activation class selection method, the best classifier on average was the ensemble random forest (RF). Regarding the highest accuracies, we find that the RLO algorithm scored $94.46 \pm 4.53$ using the first 1,000 voxels ranked by the ReliefF method. For the other combinations, SMO performed best reaching an accuracy of $97.14 \pm 2.33$ using the best 25 voxels selected by RFE.

The voxels which yielded the highest accuracy -SMO with the RFE method- are illustrated in Figure S1.A. The main activation zone is located in the visual cortex (occipital lobe). Figures S1.B, S1.C and S1.D respectively show the other three best combinations of selection algorithm and classifiers presented in Table [I] (see Supplementary Material).

\section{Discussion}

The quantitative results suggest that classification models are highly applicable in clinical scenarios. The best values for each combination show a low standard deviation (see Table II). Since these values were estimated using fair inner outer cross- 
TABLE II

AVERAGE AND MAXIMUM ACCURACY ESTIMATIONS WHEN CLASSIFYING AD AND HEALTHY INDIVIDUALS OVER 65 YEARS OLD. THE PLOTS AT THE TOP SHOW THE AVERAGE RESULTS FOR ALL PAIRS OF CLASSIFIERS AND VOXEL SELECTORS. THE BEST COMBINATION WITH THE ASSOCIATED NUMBER OF VOXELS AND ACCURACIES ARE LISTED BELOW.

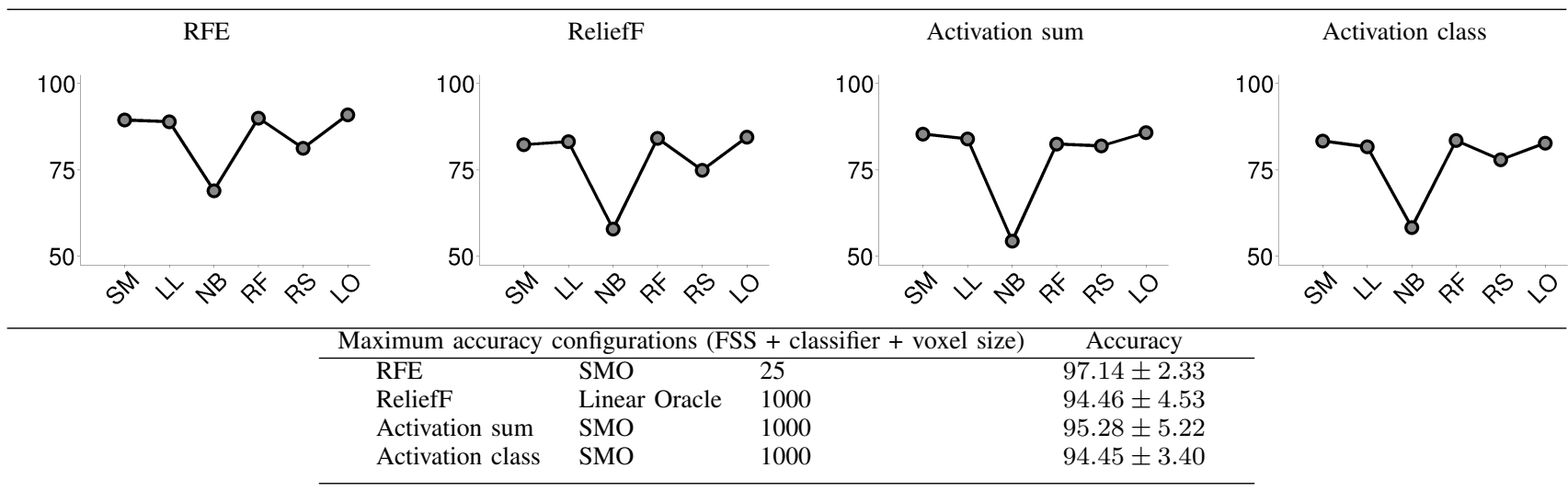

validation loops, results are not expected to be biased or overfitted [24].

Regarding the classification paradigms, three out of the four highest individual performances were for SMO. Looking at average behavior, however, an ensemble of SMO models, like the random linear oracle (RLO) is better. RLO yielded the best three average scores out of the four, proving that it has better stability and generalization properties than a single classifier. Nevertheless, SMO stands out as the single classifier best suited for classifying fMRI data profiles, as corroborated elsewhere [12].

The statistical assessment of the results also highlights the poor performance of the naïve Bayes model (see average values in Table [I]. This is numerical proof of a biologically expected phenomenon: the dependence between neighboring voxel signals. Spatially close voxels will behave in accordance with fashions as large neuronal circuits activate the whole region, and, hence, the independence assumption of the naïve Bayes model fails to mimic reality.

In terms of voxel selectors, numerical performances were similar despite their disparate mathematical roots. This suggests that data quality and separability are more relevant in the selection process than the actual algorithm. This is corroborated by the fact that three out of the best four results come from the same voxel size (1,000 voxels). The concordance in the areas to which these subsets of voxels belong is discussed from a neurological point of view below.

At first glance the most relevant voxels and their locations (Supplementary Material, Figure S1) corroborate the findings reported by [2]. However, a more detailed analysis of the selected regions unveils finer regions of potential interest for the AD research community. Table IIII summarizes the Brodmann areas containing voxels selected by the best four combinations of selectors and classifiers. Further information on all the identified regions of interest, their localization and hierarchical MNI distribution are included as Supplementary Material (Tables S1 to S4).

All the combinations of classifiers and voxel selectors concurrently resulted in a selection of Brodmann areas 4,18 and 19 [26], indicating a clear difference in the fMRI signal of these areas in demented compared to non-demented patients.

Brodmann area 4 is a primary motor cortex located in the posterior portion of the frontal lobe, which is involved in the control of movements and also contains part of the somatotopic representation of the body (e.g. [27|). In general, motor deficits affect $\mathrm{AD}$ patients at later stages [28], [29]. However, the patients analyzed in this study included different grades of dementia (five subjects with mild dementia and eight with severe dementia). Thus, the detection of the primary motor cortex as an affected area can be considered as a predictive result, which can be useful for diagnosis.

Brodmann areas 18 and 19 are both visual association areas, which are located in the occipital lobe, and they are related to the visual sensory capacity. Since visual impairment is a common symptom in AD and ageing (reviewed in [39], [40]), the results of this study with fMRI data suggest that there is possibly a specific visual alteration in demented patients.

In addition to the above brain regions selected by all the combinations of classifiers and voxel selectors, ReliefF best results (Figure S1.B) classed Brodmann area 3 as a relevant brain region when fMRI data from $\mathrm{AD}$ patients were compared to the control group. This area is also considered as primary motor cortex, and it is located in the rostral portion of the parietal lobe [26]. The selection of this brain region by the machine learning tool supports motor cortex affection in dementia.

Moreover, RFE results classed Brodmann areas 6, 17, and 30 as relevant. Results regarding area 6 , which is part of the premotor cortex located in the frontal cortex, can be attributed to the functional alteration of the motor cortex described above. Since area 17 is a visual cortex located in the occipital lobe, its relevance is possibly similar to areas 18 and 19 (discussed above). Brodmann area 30 is part of the cingulate cortex, which is part of the limbic system. Alterations of the limbic system have often been shown to be involved in AD ( [41], [42], among others).

Therefore, the selection of these brain regions by the voxel selectors and classifier ensembles pinpoints their ability as 
a diagnostic tool able to identify regions with functional alterations within the demented cohort of patients.

TABLE III

BRODMANN AREAS IDENTIFIED WITHIN THE SELECTED VOXELS BY THE BEST COMBINATIONS OF CLASSIFIERS AND VOXEL SELECTORS. VALUES BETWEEN CURLY BRACKETS REFER TO THE TOTAL NUMBER OF DIFFERENT REGIONS MAPPED BY THE SELECTED VOXELS. VALUES BETWEEN ROUND BRACKETS WITHIN THE TABLE DISPLAY HOW MANY TIMES EACH BRODMANN AREA WAS JOINTLY SELECTED.

\begin{tabular}{lllll}
\hline & $\begin{array}{l}\text { RFE, } \\
\text { SMO }\{22\}\end{array}$ & $\begin{array}{l}\text { ReliefF, } \\
\text { RLO }\{35\}\end{array}$ & $\begin{array}{l}\text { ActSum, } \\
\text { SMO }\{15\}\end{array}$ & $\begin{array}{l}\text { ActClass, } \\
\text { SMO }\{18\}\end{array}$ \\
\hline Area 3 & & & $\checkmark(1)$ & $\checkmark(1)$ \\
Area 4 & $\checkmark(1)$ & $\checkmark(1)$ & $\checkmark(1)$ & $\checkmark(1)$ \\
Area 6 & $\checkmark(1)$ & & & \\
Area 17 & $\checkmark(1)$ & & & \\
Area 18 & $\checkmark(6)$ & $\checkmark(4)$ & $\checkmark(4)$ & $\checkmark(2)$ \\
Area 19 & $\checkmark(3)$ & $\checkmark(3)$ & $\checkmark(3)$ & $\checkmark(5)$ \\
Area 30 & & $\checkmark(1)$ & & \\
\hline
\end{tabular}

\section{CONCLUSION}

Neuroimaging methods use visual rating scales, volumetric measurements, and automated methods to identify, quantify, and track anatomic evidence of AD. fMRI is a powerful method which provides information on brain activity and, thus may detect changes related to AD. fMRI is based on indirect measures of neuronal activity, which rely on a coupling of local neuronal activity to a vascular hemodynamic response. However, fMRI data are difficult to understand and interpret. Given the usefulness of data mining in medical applications [30]-[33], we used machine learning tools to detect brain regions selectively affected using the data gathered in a fMRI study of demented and non-demented patients [2].

The classic disadvantage of wrapper-based feature selections for classification is the risk of features overfitting to the classification model in use [20]. This feature overfitting translates into artificially lower error estimations if the feature search is not done with caution. If the search space is too large and the search is greedy, the risk of getting stuck in local optima is high, and the performance of the classifier will depend entirely on the quality of said features. These unwanted effects in the wrapper feature search are alleviated when the search includes a random component. This stochastic behavior is by definition embedded in some methods [34], or can be achieved by the inclusion of a random component in the search. We here made use of a two layers search -inner and outer loops- approach to diversify the intermediate search datasets. The inner search estimates the features' goodness of fit through a cross-validation split, while keeping other external cross-validation (outer loop) to compute the global error estimation [24]. This nested scheme adds more computational burden to the full process, which is already slow due to the high dimensionality of the feature space. However, this randomized wrapper presents two important advantages despite the running cost: i) it is less prone to local optima; ii) it is more likely to model features dependencies in the data.

Results from Section III were therefore discussed and contrasted across methods to ensure that the numerical performance translated into biological meaning. Our results effectively revealed that the classification of $t$-maps from fMRI data is a potential source for knowledge discovery in complex conditions such as Alzheimer's disease. Although it requires techniques for dimensionality reduction and classification, the results suggest two possible applications. First, the numerical performance achieved by the best combination of voxel selector and classifier might be implemented as a tool to help with diagnosis at the point of clinical care. Secondly, the regions containing the most relevant voxels could be considered for an in-depth neuropathological analysis to detect specific changes related to $\mathrm{AD}$.

As discussed throughout Sections III and IV both the quantitative and qualitative findings in our experiments are promising. The accuracy of the best performances is around 95\%, highlighting SVM-based classifier (SMO) and voxel selector (RFE) as the best-suited data mining approaches for analyzing this kind of data. There are no remarkable statistical differences between ensemble and single classification models, although the ensemble methods yield the best average results. This better average behavior is due to the stability gained by combining individual outputs and was previously corroborated by [12].

Spatial analysis of the voxels identified showed a high degree of concordance between brain regions, including Brodmann areas 4, 18 and 19. Moreover, other brain regions involving the cingulate cortex, the primary motor cortex and visual sensory capacity were also identified by the machine learning tools as potentially affected areas.

To conclude, since MRI voxel-based measures are related to the severity of cognitive impairment (reviewed by [35]), our results support the use of machine learning approaches as an important tool to be included in the diagnoses of AD. In addition to these quantitative approaches, several other types of clinical data are being collected by transnational efforts which aim to develop early Alzheimer diagnosis tools [36]. In the long run, all clinical information should be dynamically integrated at the point of care using advanced machine learning approaches, such as Bayesian networks [37] or multivariate regression models [38].

\section{ACKNOWLEDGMENT}

R.A. is currently supported by NIH grant R01 NS39600 from the National Institutes of Health (NIH) National Institute of Neurological Disorders and Stroke (NINDS). This work was partially supported by grants BFU2012-34963 from the Spanish Ministry of Economy and Competitiveness, and ZEN15-321663 from the Alzheimer's Association.

\section{REFERENCES}

[1] Alzheimer's Association, "2015 Alzheimer's disease facts and figures," Alzheimers Dement, vol. 11, no. 3, pp. 332-384, 2015.

[2] R. L. Buckner, A. Z. Snyder, A. L. Sanders, M. E. Raichle, and J. C. Morri, "Functional brain imaging of young, nondemented, and demented older adults," J Cogn Neurosci, vol. 12, pp. 24-34, 2000.

[3] B. Jin, A. Strasburger, S. J. Laken, F. A. Kozel, K. A. Johnson, M. S. George, and X. Lu, "Feature selection for fMRI-based deception detection," BMC Bioinform, vol. 10, no. S15, 2009.

[4] H. Melero, A. Peña-Melián, M. Ríos-Lago, G. Pajares, J. A. HernándezTamames, and J. Alvarez-Linera, "Grapheme-color synesthetes show peculiarities in their emotional brain: cortical and subcortical evidence from VBM analysis of 3D-T1 and DTI data," Exp Brain Res, vol. 227, no. 3, pp. 343-353, 2013. 
[5] S. Bray, C. Chang, and F. Hoeft, "Applications of multivariate pattern classification analyses in developmental neuroimaging of healthy and clinical populations," Front Hum Neurosci, vol. 3, no. 32, 2009.

[6] S. Klöppel, C. M. Stonnington, C. Chu, B. Draganski, R. I. Scahill, J. D. Rohrer, N. C. Fox, C. R. J. Jack, J. Ashburner, and R. S. Frackowiak, "Automatic classification of MR scans in Alzheimer's disease," Brain, vol. 131, pp. 681-689, 2008.

[7] E. E. Tripoliti, D. I. Fotiadis, M. Argyropoulou, and G. Manis, "A six stage approach for the diagnosis of the Alzheimer's disease based on fMRI data," J Biomed Inform, vol. 43, pp. 307-320, 2010.

[8] R. Armañanzas, Consensus Policies to Solve Bioinformatic Problems. LAP LAMBERT Academic Publishing, 2012.

[9] W. Penny, K. Friston, J. Ashburner, S. Kiebel, and T. Nichols, Eds., Statistical Parametric Mapping: The Analysis of Functional Brain Images. Academic Press, 2006.

[10] A. C. Evans, D. L. Collins, S. R. Mills, E. D. Brown, R. L. Kelly, and T. M. Peters, "3D statistical neuroanatomical models from 305 MRI volumes," in Proc IEEE-Nuclear Science Symposium and Medical Imaging Conference, 1993, pp. 1813-1817.

[11] G. Flandin and K. J. Friston, "Statistical parametric mapping," Scholarpedia, vol. 3, no. 4, p. 6232, 2008

[12] L. I. Kuncheva and J. J. Rodríguez, "Classifier ensembles for fMRI data analysis: An experiment," Magn Reson Imaging, vol. 28, no. 4, pp. 583-593, 2010

[13] J. C. Platt, "Fast training of support vector machines using sequential minimal optimization," in Advances in Kernel Methods, B. Schölkopf, C. J. C. Burges, and A. J. Smola, Eds. MIT Press, 1999, pp. 185-208,

[14] R. E. Fan, K. W. Chang, C. J. Hsieh, X. R. Wang, and C. J. Lin, "Liblinear: A library for large linear classification," J Mach Learn Res, vol. 9, pp. 1871-1874, 2008.

[15] H. J. George and P. Langley, "Estimating continuous distributions in bayesian classifiers," in Proc of the Eleventh Conference on Uncertainty in Artificial Intelligence. Morgan Kaufmann, 1995, pp. 338-345.

[16] L. Breiman, "Random forests," Mach Learn, vol. 45, pp. 5-32, 2001.

[17] T. K. Ho, "The random subspace method for constructing decision forests," IEEE Trans Pattern Anal Mach Intell, vol. 20, no. 8, pp. 832844, 1998.

[18] L. I. Kuncheva and J. J. Rodríguez, "Classifier ensembles with a random linear oracle," IEEE Trans Knowl Data Eng, vol. 19, no. 4, pp. 500-508, 2007.

[19] H. Liu and H. Motoda, Computational Methods of Feature Selection. Chapman and Hall/CRC Press, 2008.

[20] R. Kohavi and G. John, "Wrappers for feature subset selection," Artif Intell, vol. 97, pp. 273-324, 1997.

[21] I. Guyon, J. Weston, S. Barnhill, and V. N. Vapnik, "Gene selection for cancer classification using support vector machines," Mach Learn, vol. 46, pp. 389-422, 2001.

[22] I. Kononenko, E. Simec, and M. Robnik-Sikonja, "Overcoming the myopia of inductive learning algorithms with RELIEFF," Appl Intell, vol. 7, pp. 39-55, 1997.

[23] R. Armañanzas, B. Calvo, I. Inza, M. López-Hoyos, V. MartínezTaboada, E. Ucar, I. Bernales, A. Fullaondo, P. Larrañaga, and A. M. Zubiaga, "Microarray analysis of autoimmune diseases by machine learning procedures," IEEE Trans Inf Technol Biomed, vol. 13, no. 3, pp. 341-350, 2009.

[24] A. Statnikov, C. F. Aliferis, I. Tsamardinos, D. Hardin, and S. Levy, "A comprehensive evaluation of multicategory classification methods for microarray gene expression cancer diagnosis," Bioinform, vol. 21, no. 5, pp. 631-643, 2005.

[25] R. Armañanzas, Y. Saeys, I. Inza, M. García-Torres, C. Bielza, Y. van de Peer, and P. Larrañaga, "Peakbin selection in mass spectrometry data using a consensus approach with estimation of distribution algorithms," IEEE/ACM Trans Comput Biol Bioinform, vol. 8, no. 3, pp. 760-774, 2011.

[26] K. Brodmann, Vegleichende Lokalisationslehre der Grosshirnde, Barth, Leipzig, 1909.

[27] J. D. Meier, T. N. Aflalo, S. Kastner, and M. S. A. Graziano, "Complex organization of human primary motor cortex: A high-resolution fMRI study," J Neurophysiol, vol. 100, no. 4, pp. 1800-1812, 2008.

[28] D. Suvà, I. Favre, R. Kraftsik, M. Esteban, A. Lobrinus, and J. Miklossy, "Primary motor cortex involvement in Alzheimer disease," $J$ Neuropathol Exp Neurol, vol. 58, no. 11, pp. 1125-1134, 1999.

[29] F. Ferreri, F. Pauri, P. Pasqualetti, R. Fini, G. D. Forno, and P. M. Rossini, "Motor cortex excitability in Alzheimer's disease: A transcranial magnetic stimulation study," Ann Neurol, vol. 53, no. 1, pp. 102-108, 2003.
[30] P. Larrañaga, B. Calvo, R. Santana, C. Bielza, J. Galdiano, I. Inza, J. A. Lozano, R. Armañanzas, G. Santafé, A. Pérez, and V. Robles, "Machine learning in bioinformatics," Brief Bioinform, vol. 17, no. 1, pp. 86-112, 2006.

[31] R. Armañanzas, L. Alonso-Nanclares, J. DeFelipe-Oroquieta, A. Kastanauskaite, R. G. de Sola, J. DeFelipe, C. Bielza, and P. Larrañaga, "Machine learning approach for the outcome prediction of temporal lobe epilepsy surgery," PLoS ONE, vol. 8, no. 4, p. e62819, 2013.

[32] R. Armañanzas, C. Bielza, K. R. Chaudhuri, P. Martinez-Martin, and P. Larrañaga, "Unveiling relevant non-motor Parkinson's disease severity symptoms using a machine learning approach," Artif Intell Med, vol. 58, no. 3, pp. 195-202, 2013.

[33] A. García-Bilbao, R. Armañanzas, Z. Ispizua, B. Calvo, A. AlonsoVarona, I. Inza, P. Larrañaga, G. López Vivanco, B. Suárez-Merino, and M. Betanzos, "Identification of a biomarker panel for colorectal cancer diagnosis," BMC Cancer, vol. 12, no. 43, 2012.

[34] J. H. Holland, Adaptation in Natural and Artificial Systems. The University of Michigan Press, 1975.

[35] C. R. Jack, "Alzheimer disease: New concepts on its neurobiology and the clinical role imaging will play," Radiology, vol. 263, no. 2, pp. 344 361, 2012.

[36] P. Martinez-Martin, J. Avila, and AD Research Unit Investigators, "Alzheimer center Reina Sofia foundation: Fighting the disease and providing overall solutions," J Alzheimers Dis, vol. 21, no. 2, pp. 337$348,2010$.

[37] R. Armañanzas, P. Larrañaga, and C. Bielza, "Ensemble transcript interaction networks: A case study on Alzheimer's disease," Comput Methods Programs Biomed, vol. 108, no. 1, pp. 442-450, 2012.

[38] D. Zhang, D. Shen, and Alzheimer's Disease Neuroimaging Initiative, "Multi-modal multi-task learning for joint prediction of multiple regression and classification variables in Alzheimer's disease," Neuroimage, vol. 59, no. 2, pp. 895-907, 2012.

[39] E. Kirby, S. Bandelow, and E. Hogervorst, "Visual impairment in Alzheimers disease: A critical review," J Alzheimers Dis, vol. 21, pp. $15-34,2010$.

[40] L. M. Ward, R. T. Aitchison, M. Tawse, A. J. Simmers, and U. Shahani, "Reduced haemodynamic response in the ageing visual cortex measured by absolute fNIRS," PLOS ONE, vol. 10, no. 4, p. e0125012, 2015

[41] M. W. Hopper and F. S. Vogel, "The limbic system in Alzheimer's disease. A neuropathologic investigation," Am J Pathol, vol. 85, no. 1, pp. 1-20, 1976.

[42] H. Braak and E. Braak, "Neuropathological stageing of Alzheimerrelated changes," Acta Neuropathol, vol. 82, no. 4, pp. 239-259, 1991. 